\title{
Aspectos macroeconómicos del sector agropecuario y política agrícola en Colombia
}

\section{Macroeconomic aspects of agriculture and agricultural politic in Colombia}

\author{
Gustavo Adolfo Díaz Valencia ${ }^{1}$ \\ ${ }^{1} \mathrm{PhD}$ Ciencias Económicas, Universidad Nacional \\ Docente Investigador Universidad Santo Tomas \\ gustavodiaz@santotomas.eu.co
}

Recibido 04 Noviembre 2014, Aceptado 10 de Abril 2015

\section{RESUMEN}

Se realiza una revisión sobre los aspectos macroeconómicos del sector agropecuario y la política agrícola en Colombia, encontrándose que la producción pecuaria se desarrolla en torno a poblaciones de bovinos, porcinos, caprinos, aves, ovinos, búfalos y equinos, de ellos la bovina es la más representativa según el número de individuos, solo superado por las aves, donde, el $57 \%$ de la producción bovina corresponde carne, el $4 \%$ a leche y el $39 \%$ doble propósito. Por otro lado, se ha demostrado que el sector ganadero en Colombia es especialmente importante de cara a la mitigación del cambio climático principalmente porque el $44.9 \%$ de las emisiones de gases de efecto invernadero (GEI) del país provino de la agricultura, y el $8.9 \%$ se produjo por cambios en el uso de la tierra, y ambos valores han crecido en más del 10\% en los últimos años. Queda demostrado que los pequeños productores juegan un rol predominante en la agricultura colombiana, puesto que los hogares de la economía campesina constituyen el $12 \%$ de los hogares de Colombia, representan la mayoría de los hogares en el sector agrícola y conforman el $90 \%$ de la mano de obra agrícola, cosechan la mitad del área sembrada en cultivos, y tienen considerables partes de la ganadería. En este contexto el gobierno interviene en el sector agropecuario mediante políticas de crédito, de precios, y de comercio exterior como subsidios a la exportación y restricciones a las importaciones y exportaciones, resultando en 
un fomento de desarrollo de proyectos productivos en el campo, lo cual incentiva la generación de empleo e incrementos en el ingreso de las zonas rurales.

Palabras clave: Política agropecuaria, ganadería, PIB.

\section{ABSTRACT}

A review on the macroeconomic aspects of agriculture and agricultural policy in Colombia took place, and that livestock production is developed around populations of cattle, pigs, goats, poultry, sheep, buffaloes and horses, bovine them is the more representative in terms of the number of individuals, surpassed only by birds, where $57 \%$ of bovine meat production is $4 \%$ to $39 \%$ milk and dual purpose. Furthermore, it has been shown that the livestock sector in Colombia is especially important in the face of climate change mitigation mainly because $44.9 \%$ of emissions of greenhouse gases (GHGs) in the country came from agriculture, and $8.9 \%$ occurred due to changes in land use, and both values have grown by over $10 \%$ in recent years. It is shown that small farmers play a predominant role in Colombian agriculture, since households in the rural economy constitute $12 \%$ of households in Colombia, representing the majority of households in the agricultural sector and make up $90 \%$ of the agricultural labor, harvest half the area sown to crops, and have substantial portions of livestock. In this context the government intervenes in the agricultural sector through credit policies, pricing, and trade as export subsidies and restrictions on imports and exports, resulting in promotion of development of productive projects in the field, which encourages job creation and increased income in rural areas.

Keywords: Agricultural politic, livestock, GDP.

\section{RESUMO}

Uma revisão sobre os aspectos macroeconômicos da agricultura e da política agrícola na Colômbia teve lugar, e que a pecuária é desenvolvida em torno populações de bovinos, suínos, caprinos, aves, ovinos, búfalos e cavalos, bovinos deles é o mais representativa em termos do número de indivíduos, sendo 
superado apenas por pássaros, onde $57 \%$ da produção de carne bovina é de $4 \%$ a $39 \%$ de leite e duplo propósito. Além disso, demonstrou-se que o sector da pecuária, a Colômbia é especialmente importante em face de mitigação das mudanças climáticas, principalmente porque $44,9 \%$ das emissões de gases de efeito estufa (GEE) no país vieram de agricultura, e 8,9 \% ocorreu devido a mudanças no uso da terra, e ambos os valores aumentaram mais de $10 \%$ nos últimos anos. Mostra-se que os pequenos agricultores desempenham um papel predominante na agricultura colombiana, uma vez que as famílias na economia rural constituem $12 \%$ das famílias da Colômbia, representando a maioria dos agregados familiares no sector agrícola e representam $90 \%$ do trabalho agrícola, colheita metade da área cultivada com culturas, e tem partes substanciais do gado. Neste contexto, o governo intervém no sector agrícola, através de políticas de crédito, preços e comércio como subsídios à exportação e restrições às importações e exportações, resultando em promoção do desenvolvimento de projetos produtivos no campo, o que incentiva a criação de empregos e aumento da renda nas áreas rurais.

Palavras-chave: Política agrícola, pecuária, PIB.

\section{INTRODUCCIÓN}

El sector agropecuario es el principal generador de empleos en las zonas rurales, según el DANE en el 2013, 3.5 millones de personas trabajaron en el sector, lo que equivale al $16.9 \%$ de la población ocupada total del país, siendo el tercer sector que mayor empleo genera, después del comercial y servicios comunales (FEDESARROLLO, 2013). En lo referente a las zonas rurales, la agricultura representó un $62.6 \%$ de los ocupados que corresponde a más de 2.9 millones de empleados (FINAGRO, 2014).

Las cifras de los últimos años indican una tendencia de crecimiento del sector agropecuario, en 2012 según el Ministerio de Agricultura y Desarrollo Rural, la producción de cultivos de ciclo corto aumentó en 285.600 toneladas frente al 2011. Los productos que promovieron este incremento fueron hortalizas (57.7\%), maíz 
(46.6\%) y arroz (25.2\%), debido a las mejoras en las condiciones climáticas, especialmente en el caso del arroz y a programas de apoyo del Gobierno como el "Plan País Maíz". De igual modo los productos que mostraron un mayor crecimiento entre 2012 y 2011 fueron sorgo (56.2\%), ajonjolí (48.7\%) y soya (28.9\%) (MADR, 2011). En lo que respecta a los cultivos permanentes, en 2012 se presentó un crecimiento del 4\%, lo que implicó un aumento de 612.417 toneladas, correspondiendo $42 \%$ a caña de panela, $41 \%$ a frutales y $33 \%$ a plátano. Por su parte los productos que observaron el mayor crecimiento fueron cacao $(28.1 \%)$, tabaco negro $(23.3 \%)$ y arracacha $(8.5 \%)$. En lo correspondiente al sector pecuario (Tablas 1 y 2 ) durante este mismo periodo, el sacrificio de bovinos aumentó un $5.7 \%$, siendo superior al de porcinos $(4.9 \%)$, pollo (3.5\%), y pescado (1.4\%), mientras que la producción de camarón disminuyó un $14.1 \%$. En lo que respecta a la ganadería, la cantidad de leche aumentó en 198 millones de litros mientras que la de huevos disminuyó en 56 millones de unidades (FINAGRO, 2014).

En Colombia la producción pecuaria se desarrolla en torno a poblaciones de bovinos, porcinos, caprinos, aves, ovinos, búfalos y equinos, de ellos la bovina (Figura 1) es por mucho la más representatividad tiene según el número de individuos (Tabla 1), solo superado por las aves (Tabla 2), que dado su tamaño corporal y condiciones de manejo confinado no requieren de grandes extensiones de tierra (ICA, 2013).

Definitivamente en Colombia la ganadería es un sector productivo importante en el contexto económico y social del país, identificar sus impactos y las configuraciones territoriales que propicia se hace indispensable, sobre todo en la crisis agraria enmarcada por dos paros campesinos en menos de un año y en el actual escenario donde el gobierno nacional firma tratados de libre comercio con diversos países, y promueve intensivamente los proyectos minero energéticos, que se prevé incidirán fuertemente en los cambios del uso del suelo que históricamente tenían en Colombia, y una de esas variaciones las ha tenido la ganadería, donde se han talado los bosques para sembrar gramíneas que son la principal fuente de alimentación de los bovinos, los departamentos con más del 
4\% de participación en la producción son: Antioquia, Córdoba, Casanare, Meta, Santander, César y Caquetá (Figura 2).

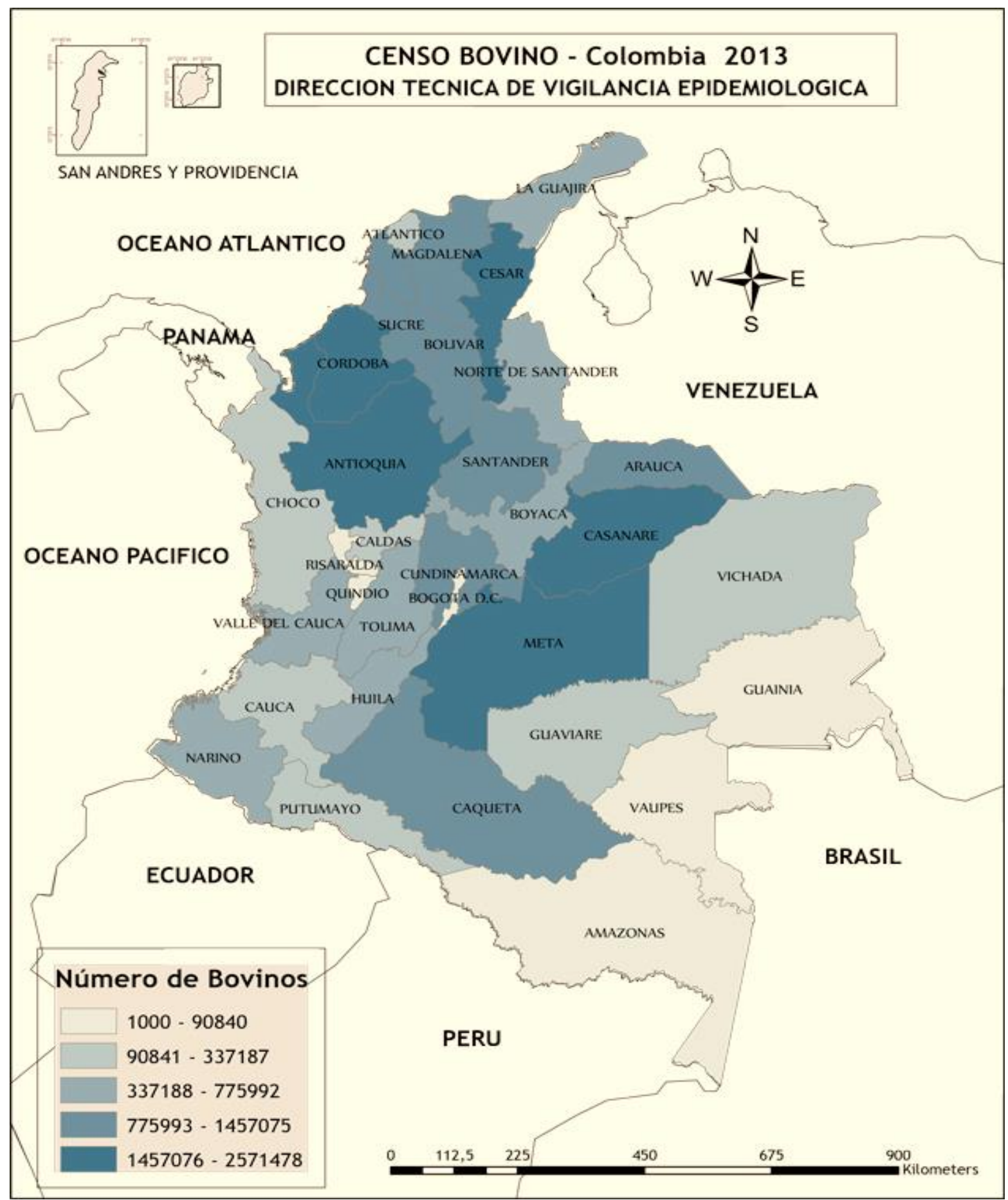

Figura 1. Censo Bovino 2013 (ICA, 2013) 
Tabla 1. Caracterización de la producción de rumiantes en Colombia, 2012 (Número de cabezas)

\begin{tabular}{|c|c|c|c|c|}
\hline Departamento & Bovinos & Ovinos & Búfalos & Caprinos \\
\hline Amazonas & 2.209 & 0 & 350 & 55 \\
\hline Antioquia & 2.571 .478 & 15.396 & 35.877 & 4.996 \\
\hline Arauca & 1.044 .750 & 14.100 & 2.345 & 1.080 \\
\hline Atlántico & 207.822 & 9.701 & 2.558 & 2.601 \\
\hline Bolívar & 790.754 & 22.359 & 10.625 & 5.475 \\
\hline Boyacá & 775.992 & 12.456 & 3.846 & 3.417 \\
\hline Caldas & 337.187 & 4.816 & 1.909 & 503 \\
\hline Caquetá & 1.338 .753 & 22.480 & 3.623 & 18.857 \\
\hline Casanare & 1.862 .540 & 2.680 & 4.750 & 12.900 \\
\hline Cauca & 266.718 & 16.034 & 316 & 3.438 \\
\hline Cesar & 1.520 .055 & 76.317 & 9.085 & 27.748 \\
\hline Choco & 125.890 & 247 & 668 & 75 \\
\hline Córdoba & 2.042.971 & 69.089 & 49.485 & 4.612 \\
\hline Cundinamarca & 1.216 .246 & 10.419 & 2.415 & 3.915 \\
\hline Distrito Capital & 19.441 & 0 & 3 & 40 \\
\hline Guainía & 3.750 & 15 & 0 & 0 \\
\hline Guaviare & 270.000 & 2.805 & 60 & 135 \\
\hline Huila & 477.447 & 5.175 & 433 & 2.061 \\
\hline La Guajira & 343.445 & 603.382 & 47 & 881.786 \\
\hline Magdalena & 1.280 .793 & 113.562 & 9.840 & 53.939 \\
\hline Meta & 1.632 .420 & 23.690 & 9.135 & 5.965 \\
\hline Nariño & 362.865 & 16.659 & 1.302 & 591 \\
\hline Norte de Santander & 473.103 & 13.136 & 1.621 & 4.344 \\
\hline Putumayo & 188.272 & 3.345 & 314 & 378 \\
\hline Quindío & 73.581 & 498 & 375 & 613 \\
\hline Risaralda & 90.840 & 411 & 323 & 284 \\
\hline San Andrés y Providencia & 1.000 & 122 & 0 & 53 \\
\hline Santander & 1.457 .075 & 45.270 & 22.085 & 44.435 \\
\hline Sucre & 856.209 & 23.599 & 10.253 & 3.985 \\
\hline Tolima & 676.981 & 42.572 & 2.412 & 2.163 \\
\hline Valle del Cauca & 480.911 & 6.079 & 1.844 & 876 \\
\hline Vaupés & 1.055 & 165 & 0 & 0 \\
\hline Vichada & 215.700 & 1.365 & 180 & 400 \\
\hline Total & 23.008 .253 & 1.177 .944 & 188.079 & 1.901 .720 \\
\hline
\end{tabular}

Fuente: Censo Agropecuario 2013 (ICA, 2013).

Los pequeños productores tienen un papel predominante puesto que los hogares de la economía campesina constituyen el $12 \%$ de las familias en Colombia, correspondiendo la mayoría al sector agrícola, quienes conforman el $90 \%$ de la mano de obra en las cosechas, donde gran parte del área sembrada para cultivos son para ganadería (12-40\%), sin embrago, la mayoría viven en condiciones de pobreza $(64 \%)$ extrema (33\%), además registran una alta informalidad en la 
posesión de sus predios y es limitado el acceso a los créditos y tecnologías (Baribbi y Spijkers, 2011).

Tabla 2. Caracterización de la producción porcina, avícola y equina en Colombia,

\begin{tabular}{|c|c|c|c|}
\hline Departamento & Porcinos & Aves & Equinos \\
\hline Amazonas & 609 & 69.740 & 50 \\
\hline Antioquia & 1.379 .875 & 10.335 .899 & 127.674 \\
\hline Arauca & 38.690 & 264.300 & 62.500 \\
\hline Atlántico & 63.599 & 4.400 .627 & 11.949 \\
\hline Bolívar & 37.249 & 1.612 .759 & 50.625 \\
\hline Boyacá & 59.519 & 2.184 .706 & 23.438 \\
\hline Caldas & 139.535 & 1.470 .393 & 39.244 \\
\hline Caquetá & 52.609 & 377.751 & 55.324 \\
\hline Casanare & 34.585 & 52.700 & 127.470 \\
\hline Cauca & 35.749 & 5.158 .579 & 82.186 \\
\hline Cesar & 31.379 & 466.535 & 56.688 \\
\hline Choco & 29.069 & 247.869 & 10.815 \\
\hline Córdoba & 141.286 & 1.466 .922 & 158.173 \\
\hline Cundinamarca & 377.965 & 28.837 .169 & 164.388 \\
\hline Distrito Capital & 1.952 & 387.450 & 943 \\
\hline Guainía & 325 & 6.050 & 55 \\
\hline Guaviare & 1.780 & 70.850 & 7.095 \\
\hline Huila & 89.499 & 1.330 .300 & 20.059 \\
\hline La Guajira & 21.250 & 114.210 & 22.440 \\
\hline Magdalena & 87.703 & 1.551 .825 & 65.756 \\
\hline Meta & 117.845 & 2.174 .300 & 63.175 \\
\hline Nariño & 140.446 & 2.557 .438 & 34.645 \\
\hline Norte de Santander & 47.060 & 1.877 .440 & 51.614 \\
\hline Putumayo & 13.761 & 429.452 & 10.490 \\
\hline Quindío & 38.498 & 6.766 .490 & 6.759 \\
\hline Risaralda & 106.599 & 3.140 .625 & 8.671 \\
\hline San Andrés y Providencia & 1.765 & 15.947 & 113 \\
\hline Santander & 80.748 & 34.995 .330 & 77.810 \\
\hline Sucre & 129.317 & 921.269 & 51.862 \\
\hline Tolima & 109.113 & 2.640 .454 & 115.505 \\
\hline Valle del Cauca & 506.654 & 40.065 .737 & 19.200 \\
\hline Vaupés & 100 & 2.600 & 25 \\
\hline Vichada & 4.015 & 25.200 & 6.680 \\
\hline Total & 3.920 .148 & 156.038 .909 & 1.533 .432 \\
\hline
\end{tabular}

Fuente: Censo Agropecuario 2013 (ICA, 2013).

La alta participación en la producción de ovinos y caprinos del departamento de La Guajira (Figura 3) se debe a que la Gobernación, entidades privadas y el SENA se unieron para trabajar en un proyecto de fortalecimiento y desarrollo competitivo del sector ovino-caprino a través de la implementación de procesos técnicos y 
empresariales. Más de 11.000 indígenas Wayúu y campesinos de este departamento se están beneficiando porque se están impartiendo programas de formación enfocados en la producción de ovinos y caprinos, procesamiento de productos cárnicos, sistemas de riego y plantas de sacrificio (ICA, 2013; Contexto Ganadero, 2013).

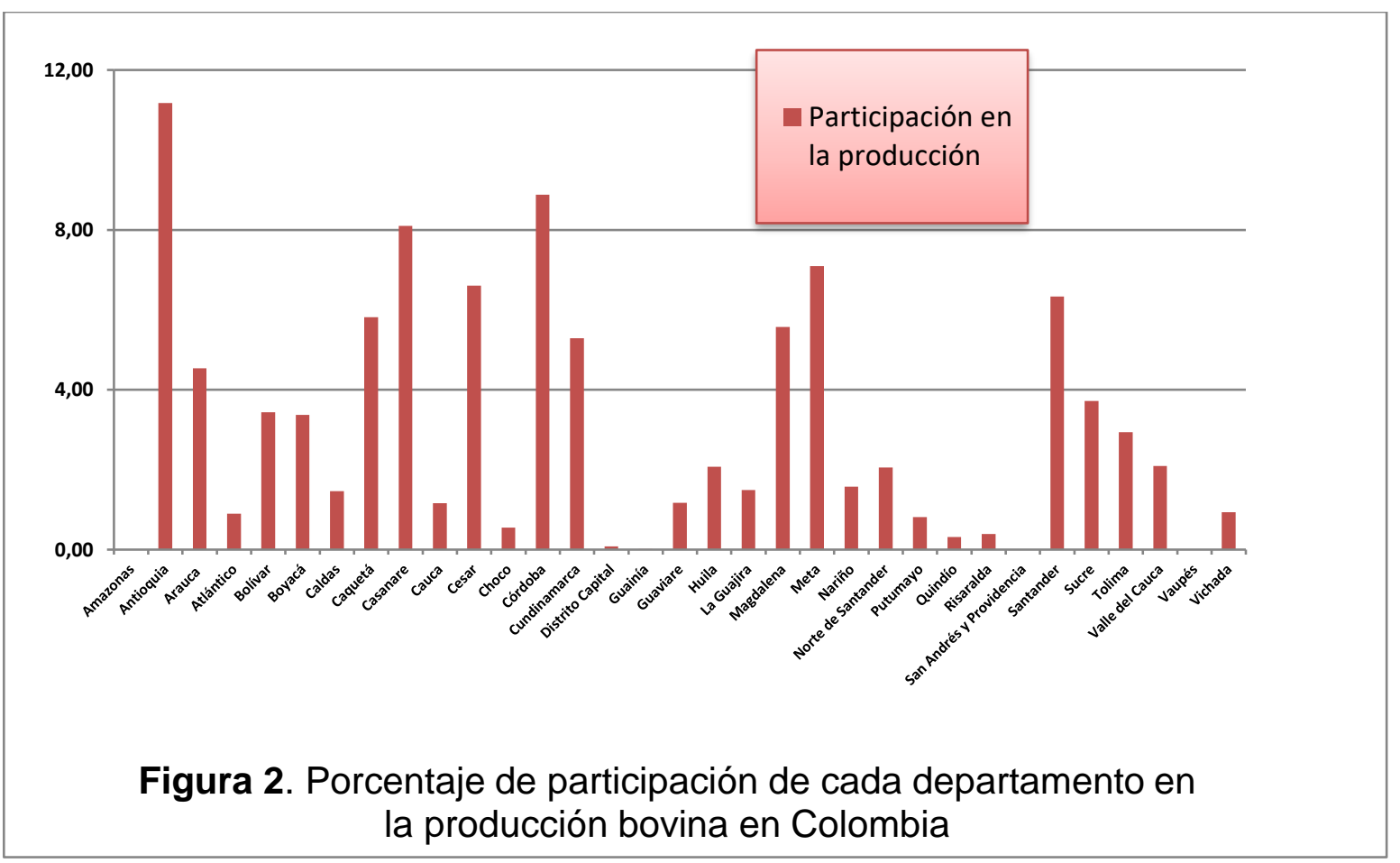

Antioquia es el mayor productor porcícola de Colombia, su participación es casi del 12\%, (Figura 4), muestra de ello es que, en el año 2012 cerca de tres millones de cerdos pasaron por los beneficiaderos, de los cuales el departamento aportó 1.379.875. Además, tiene un consumo per cápita superior a los $17 \mathrm{~kg}$, siendo el mayor consumidor de esta carne en el país, en comparación con el promedio nacional que se ubica en $5.5 \mathrm{~kg}$ per cápita, cuenta con la Asociación de Porcicultores de Colombia, constituida por un grupo de 1.400 a 1.500 productores, son los que hacen el grueso de la producción, dentro de un número de predios destinados para esta labor, que van desde los 55.000 a 65.000. De otra parte, los pequeños y medianos porcicultores están realizando alianzas en las cuales, a través de sociedades anónimas de cooperativas, han logrado fortalecerse cada día más (ICA, 2013 y Berrio, 2012). 


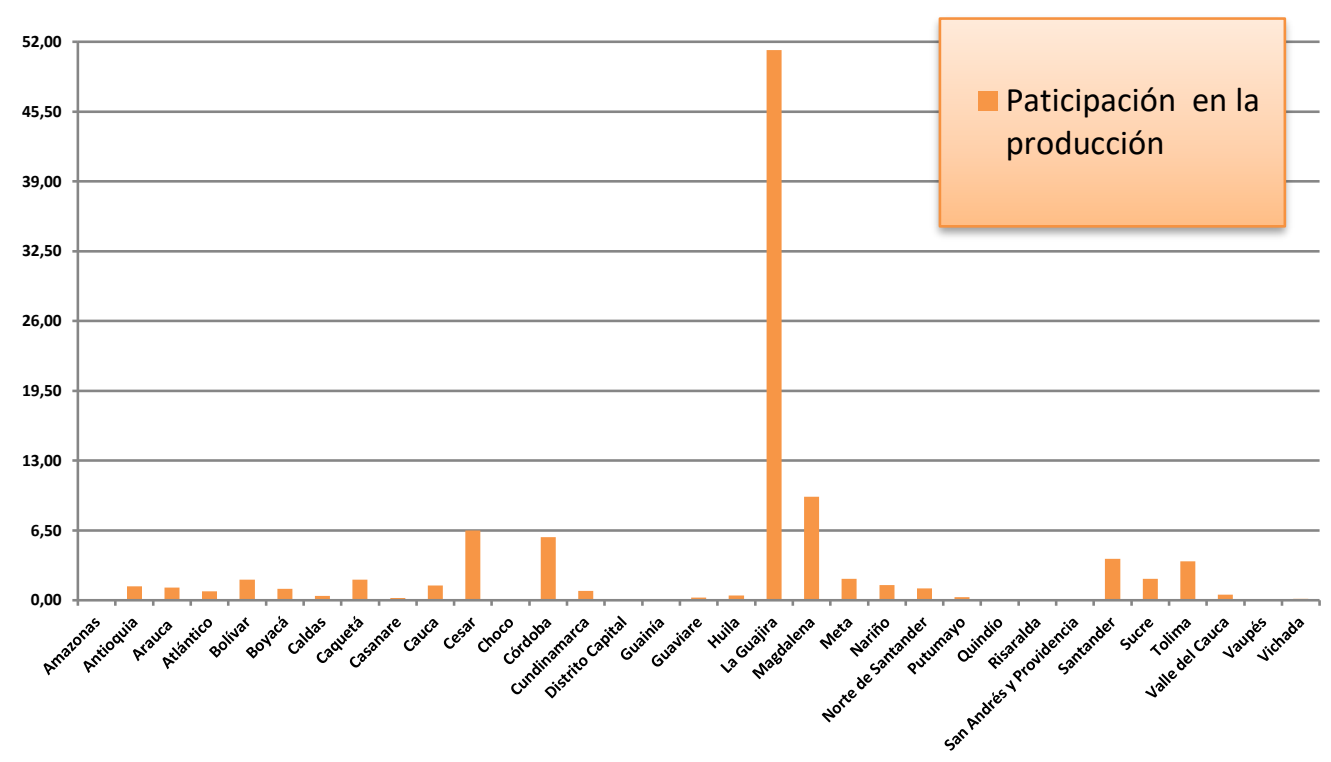

Figura 3. Porcentaje de participación de cada departamento en la producción ovina en Colombia

Los departamentos que más participan (mayor del 20\%) en la producción avícola son Valle del Cauca y Santander (Figura 5), precisamente, este sector hace parte del clúster de proteínas blancas que viene impulsando en una nueva estrategia de desarrollo de las cadenas productivas potenciales, en el 2013 se reportó que este sector se incrementó en un 9\%, cifra superior a la del año anterior. Se considera que se están dando los pasos para el Valle de Cauca lidere esta industria, no solo por parte de empresas locales, sino que otros productores de varias regiones del país vean esta región como una plataforma ideal para tener sus plantas. De otro lado la producción se está trasladando a las costas porque la industria importa el $95 \%$ del alimento para las aves y estar cerca a los puertos reduce costos y además en un futuro se facilitará las exportaciones (ICA, 2013).

Según el IGAC, (2012) de los 38 millones de hectáreas que actualmente están destinadas a la ganadería 19.3 millones tienen vocación ganadera. Sólo 5 millones de hectáreas son de pastos mejorados, y el esquema productivo vigente en este subsector se caracteriza por un uso extensivo de la tierra, tecnológicamente atrasado y con un consumo significativo de los incentivos que tiene el sector, 
especialmente crédito e ICR (Incentivo de Capitalización Rural). El mismo gremio ganadero reconoce el actual uso ineficiente $\mathrm{e}$ indeseable de las tierras colombianas y ha ofrecido como aporte a la sostenibilidad ambiental del país, devolver, antes del 2019, a la reserva forestal diez millones de hectáreas, ahora utilizadas para producción extensiva (Baribbi y Spijkers, 2011).

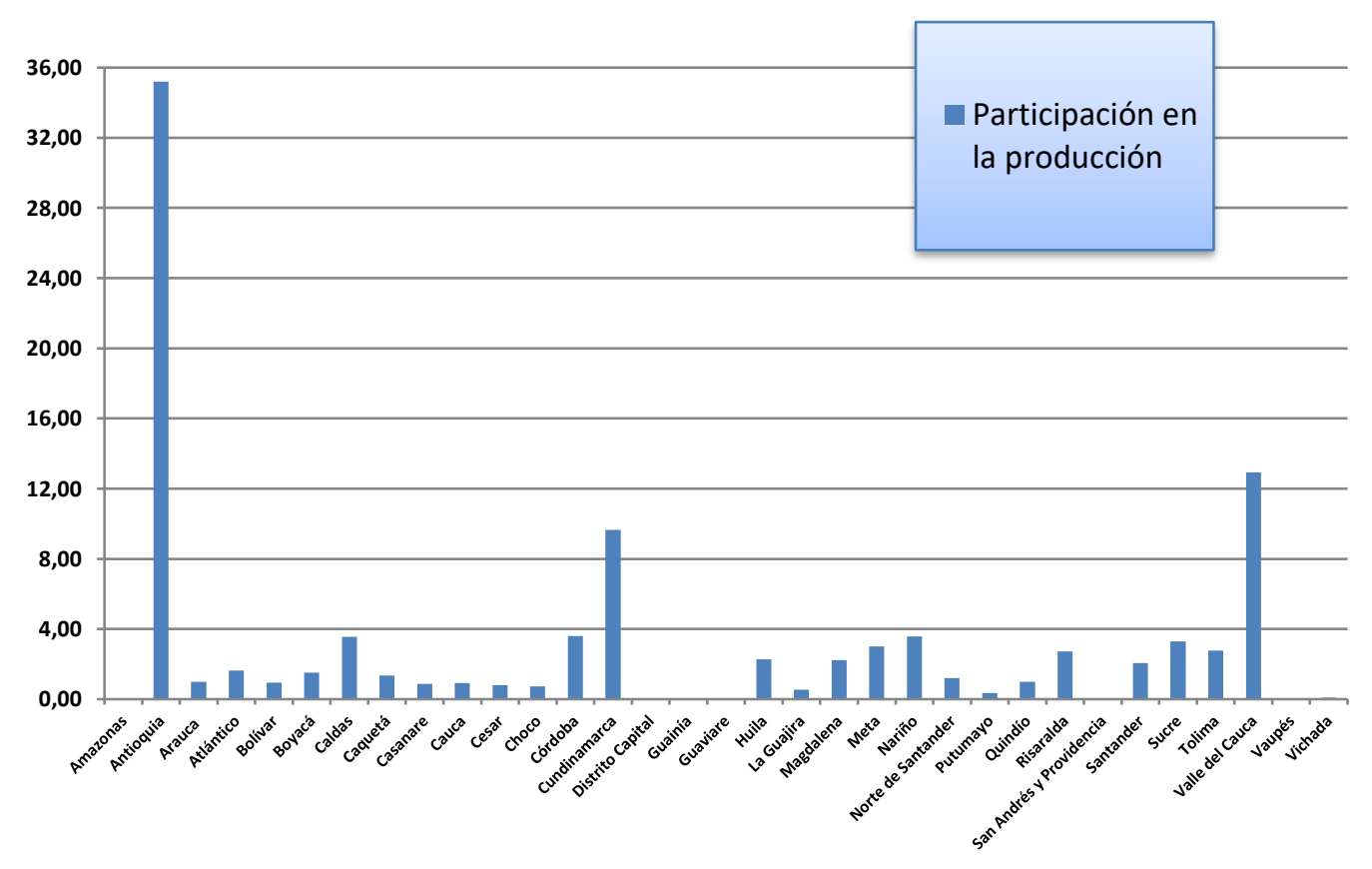

Figura 4.Porcentaje de participación de cada departamento en la producción porcina en Colombia

El Estado Colombiano y la Federación Colombiana de Ganaderos FEDEGAN han enfocado sus esfuerzos en impulsar el sector ganadero en Colombia, desde hace varios años, se han dedicado a impulsar políticas que buscan mejorar las condiciones del sector, entre las que se destaca el mejoramiento de la competitividad, hecho que se ve reflejado en la elaboración del Plan Estratégico de la Ganadería al 2019 PEGA 2019 (FEDEGAN, 2006). Desde ese momento, el seguimiento al desarrollo de los planes y a diversos indicadores se ha intensificado, con el objetivo de poder determinar cómo está la ganadería en el 
país y además se está analizando el mejoramiento a medida que se ha dado, con miras a alcanzar los objetivos del 2019.

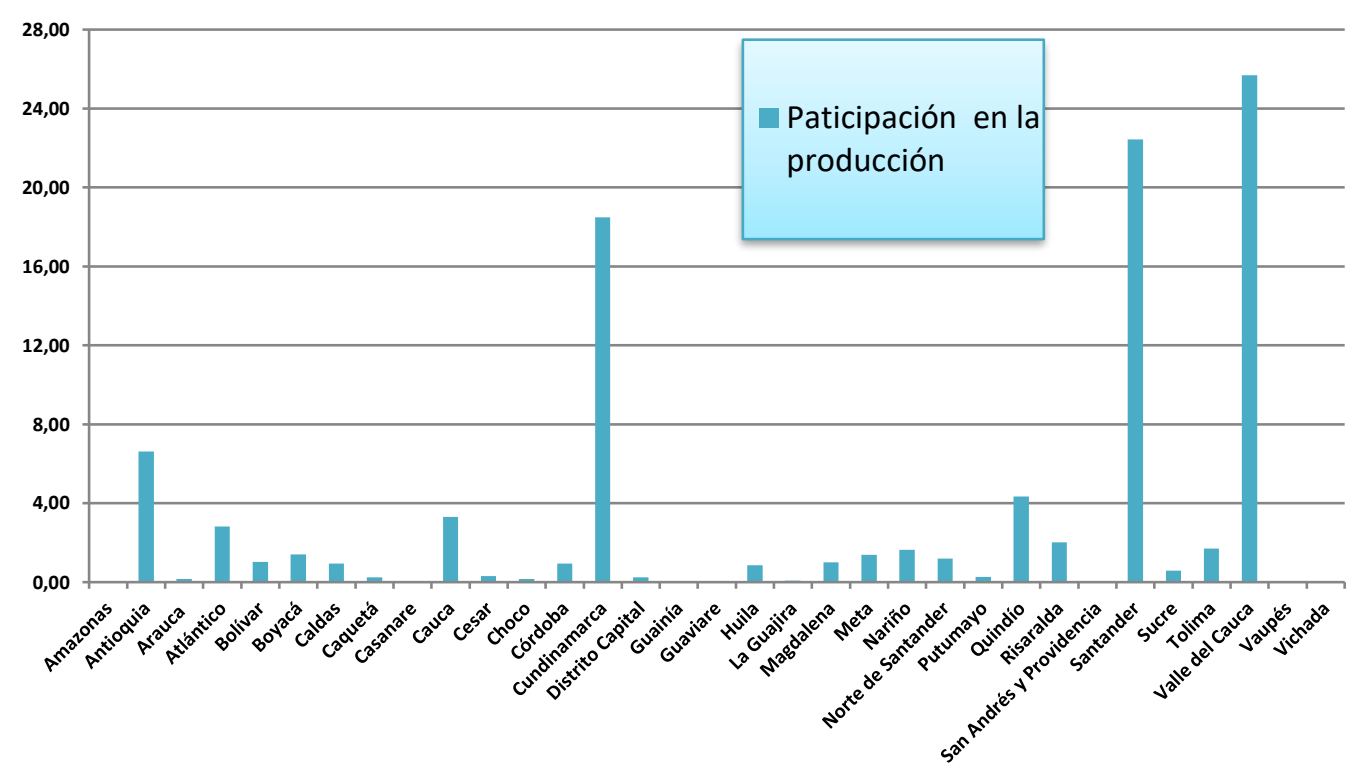

Figura 5. Porcentaje de participación de cada departamento en la producción avícola en Colombia

\section{POLÍTICAS AL SECTOR GANADERO}

El gobierno interviene en este sector ganadero mediante políticas de crédito, de precios, y de comercio exterior (subsidios a la exportación y restricciones a las importaciones y exportaciones). Las intervenciones tienen como objeto aumentar las exportaciones de carne de res y rebajar los precios internos (García, 2006), no obstante con la firma de los TLC el sector ganadero empieza a ver dificultades en la fluidez de su cadena productiva.

Definitivamente los sistemas ganaderos están afrontando en el momento grandes cambios, se empieza a observar un desplazamiento de la ganadería por la economía minera y los nuevos proyectos de inversión en cereales y biocombustibles, lo que llevará a una reubicación de este sector dentro y fuera de cada departamento de Colombia, lo que influiría radicalmente cambiando la participación productiva, expuesta en los Gráficos 1, 2, 3 y 4. 
Entre 2007 y 2011, el área utilizada en palma de aceite aumentó en 240 mil hectáreas (cerca de $40 \%$ ), especialmente en la zona oriental en donde la siembra pasó de 30 mil a 115 mil hectáreas (55\%), y en la zona central de 20 mil hectáreas se pasó a 87 mil, hectáreas (FEDEGAN, 2013).

Según Naciones Unidas el sector ganadero en Colombia es especialmente importante en la mitigación del cambio climático principalmente porque el $44.9 \%$ de las emisiones de gases de efecto invernadero (GEI) del país provino de la agricultura, y el $8.9 \%$ se produjo por variaciones en el uso de la tierra y forestería, y ambos valores han crecido en más del $10 \%$ en los últimos años (Convención Marco de las Naciones Unidas sobre el Cambio Climático, UNFCCC, 2009), y más allá de eso, tiene una alta tasa de expansión (especialmente la ganadería) y por tanto de deforestación, ocupando un porcentaje significativo del área nacional; además se cuenta con la potencial producción de biocombustibles; y las tecnologías implementadas podrían resultar en un aumento sustancial en la captura de carbono dado que la actividad agropecuaria se halla en directa interacción con el suelo, y por tanto tiene el potencial para realizar con el fin de secuestrar carbono derivado de las actividades agropecuarias racionales y amigables con el medio ambiente (PNUD, 2012).

\section{PERSPERCTIVAS DEL INVENTARIO GANADERO}

Las estimaciones del comportamiento de los sistemas bovinos colombianos en el corto plazo son: alta participación de hembras en el sacrificio y desestímulos que trae la situación actual como altos costos de los insumos, bajo precio del litro de leche cruda, importaciones de leche y derivados lácteos, entre otros (FEDEGAN, 2013). Estos dos factores afectan directamente la tasa de reposición, como se mencionó anteriormente, sin embargo, el incremento en el precio del ganado en pie como resultado del mejoramiento de las exportaciones de carne y consumo doméstico de este producto activarán la producción ganadera en el mediano plazo; en ese sentido 2013 y 2014 muestran también bajas en el inventario ganadero, y se proyecta una recuperación del hato a partir de 2015. De otro lado, se espera mayor dinamismo en los flujos regionales de ganado como 
consecuencia del desplazamiento que hace la economía minera a la producción pecuaria, los nuevos proyectos de inversión en cereales y biocombustibles, lo que llevará a una reubicación de la ganadería dentro y fuera de los departamental (FEDEGAN, 2013).

En línea con la propuesta está el proyecto llamado "Ganadería Colombiana Sostenible" basado en el proyecto previo "Integrated Silvopastoral Approaches to Ecosystem Management Project in Colombia, Costa Rica and Nicaragua" (Sistemas Silvopastoriles Integrales para el Manejo de Ecosistemas en Colombia, Costa Rica y Nicaragua) ejecutado por la fundación GEF (Global Environment Facility) y el Banco Mundial en 2008.

El proyecto de "Ganadería colombiana Sostenible" es una propuesta conjunta de la Federación de Ganaderos (FEDEGAN), FINAGRO (Fondo para el Financiamiento del sector Agropecuario), TECNIGAN (Técnicas Ganaderas), TNC (The Nature Conservancy), el MADR (Ministerio de Agricultura y Desarrollo Rural), el MAVDT (Ministerio de Ambiente, Vivienda y Desarrollo Territorial), y otros actores de relevancia para el sector que implementarán la conversión de tierras ganaderas degradadas y de pasturas mejoradas con laboreo intensivo, en sistemas silvopastoriles, mediante el uso de créditos de conversión, pagos por servicios ambientales, e incentivos adicionales de condonación de créditos por realización de prácticas de reforestación. Es decir, la ganadería en Colombia empezará a reconfigurarse hacia la implementación de la economía verde propuesta por Naciones Unidas que como se sabe va en horizonte de ampliar los límites del capitalismo (mercantilización de la naturaleza) a través del "enverdecimiento" de todos los sectores productivo (PNUD, 2012).

\section{CONSUMO DE CARNE DE RES}

El consumo de carne de res en el país ha crecido a partir de 2009, debido al aumento del ingreso medio de los hogares (FEDEGAN, 2013). De acuerdo al informe de pobreza monetaria y desigualdad del DANE, el ingreso promedio per cápita de la unidad de gasto de la población colombiana creció cerca de $24 \%$ entre 
los años 2009 y 2010. Por su parte, el consumo de los hogares en el mismo periodo presentó una tasa promedio de crecimiento anual del $4 \%$. Cabe recordar que la carne de res es un bien elástico ante cambios en el nivel de ingresos, es decir, a mayor nivel de ingresos de una persona aumenta su consumo por res (Baena y Montoya, 2010), para Colombia la elasticidad ingreso es de $1.38 \%$

La carne de res es la segunda carne más consumida en Colombia, según FEDEGAN, en 2013 se consumieron 20 kg per cápita, presentando una caída del $3.6 \%$. Sin embargo, la FAO, (2012) estima un crecimiento del consumo de carne bovina de un $58 \%$ entre 2010 y 2050 , además de ser un producto con una amplia oferta en el país, es una fuente considerable de proteínas (22.33\%), lo que la hace fundamental para la seguridad alimentaria del país.

La producción ganadera es el principal componente del producto interno bruto (PIB) pecuario, pese a tener una caída del $0.5 \%$ en 2013, su peso relativo en el sector es del 31\%. Según el MADR, en 2012 la extracción total registrada de ganado bovino fue superior a 4 millones de cabezas, presentando un crecimiento del 5.7\% (MADR, 2013), además Colombia cuenta con el cuarto inventario de América Latina y el décimo séptimo a nivel mundial (22.6 millones), siendo Antioquía y Córdoba los departamentos con mayor inventario, de igual forma tiene un crecimiento potencial superior al $20 \%$ y más de 700 mil colombianos viven del sector.

\section{FINANCIAMIENTO DEL SECTOR AGROPECUARIO}

Se está fomenta el desarrollo de proyectos productivos en el campo, lo cual incentiva la generación de empleo e incrementos en el ingreso de las zonas rurales (MADR y MHCP, 2006). El financiamiento al sector rural, ha presentado una evolución favorable en los últimos años, como lo dejan ver indicadores de acceso y profundización. El sector donde se presentó el mayor incremento en el valor del crédito en 2013 fue el avícola (183 mil millones). Si bien la ganadería continuó con la tendencia a obtener alto valor de crédito, con una participación del $18.8 \%$ del total, dentro de los productos agrícolas, la mayor participación la 
continúa teniendo el arroz (9.9\%), sin embargo, el producto que presentó el mayor crecimiento fue tabaco (553.6\%) (DDRS y FINAGRO, 2014).

Dentro de las medidas se destacan por su alcance y beneficio para los pequeños y medianos productores del país: el Proyecto de Ley 134C-140S, la reactivación y ampliación del programa de compra de cartera FONSA 2014, la aprobación del nuevo modelo de gestión del Fondo Agropecuario de Garantías, y la implementación de dos nuevos instrumentos financieros: microcrédito y Factoring (FINAGRO, 2014).

\section{Proyecto de Ley 134c-140s}

Fortalece el esquema de financiamiento para la reactivación del sector agropecuario, los principales aspectos incorporados en la Ley son: impulso al microcrédito a través de la creación del Fondo de Microfinanzas; agilidad en la aprobación de los créditos mediante la eliminación el trámite de calificación previa; destinación de los recursos del crédito agropecuario para actividades de transformación de productos del sector; mayor cobertura de riesgos agropecuarios a través de la ampliación del objeto del Fondo Nacional de Riesgos Agropecuarios; posibilidad de que dicho Fondo financie estudios sobre los productos agropecuarios a fin de fomentar la oferta de seguros; fortalecimiento del fondo agropecuario de garantías (FAG) en la recuperación de sus cartera, la deducción de provisiones y reservas para el pago de impuestos, y la simplificación de causales de no pago; fortalecimiento de FINAGRO; se reconfirma la naturaleza jurídica de FINAGRO como entidad de economía mixta; ampliación de los alivios a los actuales deudores del fondo de solidaridad agropecuario (FONSA) y del programa nacional de reactivación agropecuaria (PRAN); ampliación de los objetivos del FONSA, situaciones de crisis objeto del FONSA y extensión de plazos para deudores del PRAN y el FONSA; fortalecimiento de CORPOICA (FINAGRO, 2014). El acceso al financiamiento por parte del sector rural ha aumentado, en 2013 los usuarios de crédito registrado en FINAGRO representaron el $38 \%$ de los productores del sector en el país, lo cual implica un aumento de 27 puntos porcentuales desde 2003. 


\section{CRISIS DEL SECTOR LÁCTEO}

En los últimos cinco años, el eslabón primario del sector lácteo colombiano ha sufrido las consecuencias de una política de apertura en el agro colombiano y la carencia de políticas públicas que ayuden a superar los problemas estructurales de los productores primarios, factores que no permiten que este sector sea competitivo en el entorno actual (FEDEGAN, 2013).

La vigencia de los TLC fomentó la entrada de importaciones de productos lácteos, principalmente en 2012, cuando ingresaron al mercado colombiano más de 33.000 toneladas de productos lácteos en especial de leche en polvo (67\%). Esto llevó y aceleró la reducción del precio pagado al ganadero, el cual no compensa el incremento de los costos de los insumos agropecuarios durante estos años (FEDEGAN, 2013).

Durante los años 2009 al 2011 las actividades agropecuarias se vieron afectados por los fenómenos climáticos que en algunos casos ocasionaron pérdidas en más del $60 \%$ de algunos predios al inundarse estos (BID y CEPAL, 2012). La anterior situación ha llevado a que la rentabilidad de la actividad ganadera se vea afectada lo que ha ocasionado que algunos productores tomen la fuerte decisión de cambiar de actividad, en el mejor de los casos se cambian a producción de carne, en el peor abandonan la ganadería.

\section{CARACTERÍSTICAS DE LOS LLANOS ORIENTALES}

Es una región que ocupa una extensión de 35.010.300 hectáreas, su conformación fisiográficas la hace importante en el contexto nacional por su variedad de ecosistemas, el aporte del $32 \%$ a las reservas de agua del país y su potencial agropecuario y minero-energético, por lo tanto, su ubicación estratégica eleva su potencial y competitividad (MADR, 2013).

La zona está conformada principalmente por los departamentos de Meta, Casanare, Arauca y Vichada, y tiene una fuerte integración económica y cultural con los departamentos cercanos de Guaviare, Guainía y Vaupés, registrando una 
población de 1.5 millones de habitantes, con una mayor participación del Meta con cerca de 900 mil habitantes, de la cual casi la mitad se concentra en su capital, Villavicencio (DNP, 2011).

\section{Potencialidades}

Posee suelos con aptitud forestal altamente productiva, siendo el epicentro de este desarrollo en el país, puesto que dispone de ocho millones de hectáreas para establecer árboles sin necesidad de desplazar ningún otro cultivo, ni intervenir bosques nativos, por lo tanto se hace atractiva para la inversión de proyectos de reforestación comercial de gran envergadura. Así mismo, la riqueza de recursos naturales, tales como agua, tierras planas, condiciones agroclimáticas excepcionales de pluviosidad, luminosidad y brillo solar, que permiten una importante diversidad de actividades productivas (MADR, 2013). Es la región de interés para desarrollar megaproyectos agroindustriales, sociales y ambientales, cuya topografía posibilita la mecanización de todas las actividades de siembra, manejo y cosecha, además hay riqueza petrolera e ingresos provenientes de las regalías.

\section{Restricciones}

La región tiene una estacionalidad en la oferta de agua, lo cual impone limitaciones ambientales, debido a la fragilidad de sus ecosistemas. Por otro lado, no se puede negar que falta mayor acervo tecnológico, hay escasa formalización de la propiedad y unidades de agricultura familiar con tamaños no adecuados para grandes emprendimientos (MADR, 2013). De manera similar, falta claridad sobre el derecho de propiedad de la tierra, encontrándose el Catastro desactualizado, donde hay tierra titulada pero no registrada, tierras baldías ocupadas y desocupadas, o que son trabajadas por terceros con los de resguardos indígenas, deficiente infraestructura productiva (riego, almacenamiento) y de transporte, agravándose la situación por una insuficiente atención social en lo relacionado con salud, educación, saneamiento básico y vivienda. 


\section{Producción pecuaria}

La región ha desarrollado tradicionalmente una ganadería extensiva de carne, donde el inventario ganadero asciende aproximadamente a 4.6 millones de cabezas de ganado, y la orientación del hato es: 50\% carne, 39\% doble propósito, y $10 \%$ leche (MADR, 2003).

\section{Lineamientos estratégicos para impulsar el desarrollo rural}

Debe ser un punto clave la restitución de tierras, y consecuentemente su titulación, poniendo énfasis en la vivienda de interés social rural. Así mismo, es necesaria una fuerte inversión en ciencia y tecnología, brindando asistencia subsidiada a los pequeños y productores. Además de los incentivos financieros que lo pueden apoyar mediante el Incentivo a la Capitalización Rural (ICR), Línea Especial de Crédito (LEC), Certificado al Incentivo Forestal (CIF), es importante la firma de convenios con el CIAT, (2011 y 2012) para innovación, desarrollo y validación tecnológica (modelos de sistemas de producción ganadera, agrícola y forestal y manejo integral del suelo, entre otros), identificándose especies promisorias como: eucalipto, acacia, pino flora, nativas y materiales forrajeros), para la elaboración de modelos productivos con enfoque territorial. La investigación y el desarrollo tecnológico en la altillanura llevan más de tres décadas, con el aporte de entidades nacionales e internacionales como ICA, CORPOICA, CIMMYT, CIAT, Fedearroz, Gaviotas, Universidad de Los Llanos, Universidad Nacional, Yamato y Embrapa.

\section{UNIDAD DE RESTITUCIÓN DE TIERRAS}

Más de 22 mil hectáreas se han restituido a cerca de 400 personas víctimas de abandono y despojo forzado de tierras en el Meta, son en total 39 zonas de intervención ubicadas en 18 de los 29 municipios que integran este departamento, en donde la Unidad de Restitución de Tierras ha liderado su labor para lograr que las familias recuperen los predios que creían perdidos por la incidencia de los grupos armados al margen de la ley. A la fecha, esta política ha llegado a los municipios de: Puerto Gaitán, Puerto López, Acacias, El Castillo, El Dorado, 
Mapiripán, Cabuyaro, Villavicencio, Restrepo, San Carlos de Guaroa, Lejanías, Puerto Lleras, Castilla la Nueva, Fuente de Oro, Cumaral, Guamal, Barranca de Upía y Cubaral; obteniendo como resultado más de 348 demandas puestas en manos de los jueces y magistrados designados (URT, 2015).

Son en total 55 sentencias las que se han proferido sobre predios ubicados en las jurisdicciones de Acacías, Cumaral, El Dorado, Granada, Puerto Gaitán, Puerto López, Restrepo y Villavicencio. Tierras en las que se han invertido más 574 millones de pesos para emprender proyectos productivos, brindando herramientas de reparación integral a quienes fueron víctimas de la violencia (URT, 2015).

En esta parte del país la Corporación Colombia Internacional (CCl) viene impulsando la formalización y el fortalecimiento de organizaciones rurales, para generar empleo e ingresos estables a los pobladores del campo. Es así como bajo diferentes programas y estrategias se han beneficiado más de 5.200 familias en esta región.

Igualmente el Ministerio de Agricultura y la $\mathrm{CCl}$, vienen fomentando la creación de microempresas en el campo, a través del Programa Oportunidades Rurales se capacitaron a 320 familias de 14 organizaciones Mypes, ubicadas en los departamentos de Arauca, Guainía, Meta, Guaviare, Casanare y Vaupés. Estos beneficiarios recibieron recursos por $\$ 560$ millones de pesos para respaldar sus iniciativas productivas y planes de inversión (MADR, 2014).

\section{CONCLUSIONES}

En Colombia la producción pecuaria se desarrolla en torno a poblaciones de bovinos, porcinos, caprinos, aves, ovinos, búfalos y equinos, de ellos la bovina es por mucho la más representativa según el número de individuos, solo superado por las aves, que dado su tamaño corporal y condiciones de manejo confinado no requieren de grandes extensiones de tierra. En cuanto a la caracterización de la ganadería, el $57 \%$ de la producción bovina corresponde carne, el $4 \%$ a leche y el $39 \%$ doble propósito. 
Los pequeños productores juegan un papel predominante en la agricultura colombiana, los hogares de la economía campesina constituyen el $12 \%$ del total de Colombia, representan la mayoría en el sector agrícola y conforman el $90 \%$ de la mano de obra agrícola, cosechan la mitad del área sembrada en cultivos, y tienen considerables partes de la ganadería, siendo los departamentos más productores: Antioquia, Cundinamarca, Santander, Valle del Cauca, Córdoba, Magdalena, Meta, Casanare, sobresaliendo en la ovinocultura La Guajira.

El sector ganadero en Colombia es especialmente importante puesto que puede participar activamente en la mitigación del cambio climático principalmente porque el $44.9 \%$ de las emisiones de gases de efecto invernadero (GEI) del país provienen de la agricultura, y el $8.9 \%$ se generan por variaciones en el uso de la tierra y forestería, es de anotar que ambos valores han crecido en más del $10 \%$ en los últimos años.

\section{REFERENCIAS BIBLIOGRÁFICAS}

1. Baena LJ, Montoya LM. Determinantes del precio al consumidor de la carne bovina en la ciudad de Medellín, Antioquia: 1998-2008. Trabajo de grado, Economista. Universidad EAFIT. 39 p. 2010.

2. BID (Banco Interamericano de Desarrollo), CEPAL (Comisión económica para América Latina y el Caribe). Valoración de daños y pérdidas, Ola invernal en Colombia 2010-2011. 240 p. 2012.

3. Baribbi A, Spijkers P. Campesinos tierra y desarrollo rural. Reflexiones desde la experiencia del Tercer Laboratorio de Paz. Acción Social y Unión Europea. 28 p. 2011.

4. Berrío M. Antioquia Porcícola. 2012. Disponible En: http://www.elmundo.com/portal/noticias/economia/antioquia porcicola.php\#.V Up5rl5 NBC

5. Contexto Ganadero. La Guajira quiere modernizar y fortalecer la producción ovina. 2013. Disponible En: http://contextoganadero.com/regiones/la-guajiraquiere-modernizar-y-fortalecer-la-produccion-ovina

6. DDRS (Dirección de Desarrollo Rural Sostenible), FINAGRO (Fondo para el Financiamiento de Sector Agropecuario). Misión para la transformación del campo. Sistema nacional de crédito agropecuario, Propuesta de reforma, $63 \mathrm{p}$. 2014.

7. DNP (Departamento Nacional de Planeación). Plan nacional de Desarrollo 2010-2014. $541 \quad$ p. $2011 . \quad$ Disponible En: https://sinergia.dnp.gov.co/SISMEG/Archivos/PND20102014\%20Tomo\%201\%20CD.pdf 
8. FAO (Organización de las Naciones Unidas para la Alimentación y la Agricultura). Ganadería mundial 2011 - La ganadería en la seguridad alimentaria, Roma. 2012.

9. FEDEGAN (Federación Nacional de Ganaderos), FNG (Fondo Nacional ganadero). Análisis del inventario ganadero colombiano. Comportamientos y Variables explicativas, 22 p. 2013.

10. FEDEGAN (Federación Nacional de Ganaderos). Plan Estratégico de la ganadería colombiana 2019. 272 p. 2006.

11. FEDESARROLLO (Fundación para educación superior y el desarrollo), SAC (Sociedad de Agricultores de Colombia), INCODER (Instituto Colombiano de Desarrollo Rural), FINAGRO (Fondo para el financiamiento del sector agropecuario), Banco Agrario. Políticas para el desarrollo de la agricultura en Colombia. Sociedad de Agricultores de Colombia. La Imprenta Editores S.A. 234 p. 2013.

12. FINAGRO (Fondo para el Financiamiento de Sector Agropecuario). Perspectivas del sector agropecuario. 27 p. 2014. Disponible En: https://www.finagro.com.co/sites/default/files/2014 0909 perspectivas agrop ecuarias.pdf

13. FINAGRO (Fondo para el Financiamiento de Sector Agropecuario). Financiamiento del sector agropecuario se fortalece. 2014. Disponible En: https://www.finagro.com.co/noticias/financiamiento-del-sector-agrario-sefortalece

14. FINAGRO (Fondo para el Financiamiento de Sector Agropecuario). 2014. Sistemas de garantías para el agro se fortalece. Disponible En: https://www.finagro.com.co/noticias/sistema-de-garant\%C3\%ADas-para-elagro-se-fortalece

15. ICA (Instituto colombiano agropecuario ICA. 2013. Censo Nacional Agropecuario. 2013. Disponible En: http://www.ica.gov.co

16. IDEAM (Instituto de Hidrología, Meteorología y Estudios Ambientales). 2001. Primera Comunicación Nacional ante la Convención Marco de las Naciones Unidas sobre el Cambio Climático. Bogotá. 305 pp.

17. IGAC (Instituto Geográfico Agustín Codazzi) y CORPOICA (Corporación Colombiana de Investigación Agropecuaria). 2001. Panorama sobre el uso y ocupación actual de las tierras en Colombia. Bogotá. 136 p.

18. IGAC (Instituto Geográfico Agustín Codazzi). Atlas de la distribución de la propiedad rural en Colombia / El Instituto. -- Bogotá: Imprenta Nacional de Colombia, 540 p. 2012.

19. IGAC (Instituto Geográfico Agustín Codazzi). Clasificación del uso actual de las tierras. Subdirección de Agrología, Bogotá D.C. Metodología. 54 p. 1998.

20. García J. Las políticas económicas y el sector ganadero en Colombia: 19501977. Cuadernos de Historia Económica y Empresarial. Cartagena. Banco de la República. 91 p. 2006.

21. MADR (Ministerio de Agricultura y Desarrollo Rural). Llanos orientales, retos y oportunidades. Política para el sector agropecuario y desarrollo rural regional, 24 p. 2014. Disponible En: http://www.asorinoquia.org/malocas2013/Dia2.4.pdf 
22. MADR (Ministerio de Agricultura y Desarrollo Rural). Anuario estadístico del sector agropecuario 2012: resultados evaluaciones agropecuarias municipales. Evaluaciones Agropecuarias Municipales. Bogotá. 2013.

23. MADR (Ministerio de Agricultura y Desarrollo Rural). Plan país maíz, cadenas productivas. $\quad 18 \quad$ p. $2011 . \quad$ Disponible http://www.fenalce.org/archivos/Plan P M.pdf

24. MADR (Ministerio de Agricultura y Desarrollo Rural). Encuesta Nacional Agropecuaria 2009. Bogotá. 2009.

25. MADR (Ministerio de Agricultura y Desarrollo Rural), MHCP (Ministerio de Hacienda y Crédito Público). Concepto favorable a la nación para contratar un empréstito externo con el fondo internacional de desarrollo agrícola (FIDA) por un valor de hasta US \$20 millones o su equivalente en otras monedas, para financiar parcialmente el programa "desarrollo de las oportunidades de inversión y capitalización de los activos de las microempresas rurales. Documento Conpes, 15 p. 2006.

26. MADR (Ministerio de Agricultura y Desarrollo Rural). Situación de los recursos zoogenéticos en Colombia. 120 p. 2003. Disponible En: ftp://ftp.fao.org/docrep/fao/011/a1250f/annexes/CountryReports/Colombia.pdf

27. Nieto PR, Rey P. Las autodefensas y el paramilitarismo en Colombia (19642006). Revista Confines, 4 (7): 43-52. 2008.

28. Parra R. Desarrollo rural, clave para la paz en Colombia. CIAT. 2013. Disponibles En: http://dapa.ciat.cgiar.org/politicas-de-desarrollo-rural-clavespara-la-paz-en-colombia/

29. PNUD (Programa de la Naciones Unidas para el Desarrollo). Documento de discusión nacional acerca de los asuntos claves en el análisis del sector agricultura (Mitigación). 2012. Disponible En: http://www.pnud.org.co/

30. URT (Unidad de Restitución de Tierras). Informe final de gestión - Plan de acción a 31 de Diciembre de 2015. 53 p. 2015. Disponible En: https://www.restituciondetierras.gov.co/documents/10184/447616/GI-FO08+INFORME+DE+GESTION+TRIMESTRAL+V2.1.pdf/70384f74-b8ea-4301$\underline{\text { 9d51-40b935d2d4ca }}$ 